\title{
Sequence and structural features of binding site residues in protein-protein complexes: comparison with protein-nucleic acid complexes
}

\author{
M Michael Gromiha ${ }^{1,2^{*}}$, N Saranya ${ }^{3}$, S Selvaraj ${ }^{3}$, B Jayaram ${ }^{4}$, Kazuhiko Fukui ${ }^{2}$ \\ From International Workshop on Computational Proteomics \\ Hong Kong, China. 18-21 December 2010
}

\begin{abstract}
Background: Protein-protein interactions are important for several cellular processes. Understanding the mechanism of protein-protein recognition and predicting the binding sites in protein-protein complexes are long standing goals in molecular and computational biology.

Methods: We have developed an energy based approach for identifying the binding site residues in proteinprotein complexes. The binding site residues have been analyzed with sequence and structure based parameters such as binding propensity, neighboring residues in the vicinity of binding sites, conservation score and conformational switching.

Results: We observed that the binding propensities of amino acid residues are specific for protein-protein complexes. Further, typical dipeptides and tripeptides showed high preference for binding, which is unique to protein-protein complexes. Most of the binding site residues are highly conserved among homologous sequences. Our analysis showed that $7 \%$ of residues changed their conformations upon protein-protein complex formation and it is $9.2 \%$ and $6.6 \%$ in the binding and non-binding sites, respectively. Specifically, the residues Glu, Lys, Leu and Ser changed their conformation from coil to helix/strand and from helix to coil/strand. Leu, Ser, Thr and Val prefer to change their conformation from strand to coil/helix.
\end{abstract}

Conclusions: The results obtained in this study will be helpful for understanding and predicting the binding sites in protein-protein complexes.

\section{Background}

Protein-protein interactions are important for most of the cellular processes in life. Hence, understanding the mechanism of protein-protein recognition at molecular level is of practical interest and has direct applications to functional genomics. Unravelling the mechanisms of protein-protein recognition is a fundamental problem, which would aid in function prediction and drug design.

The availability of structures of numerous protein-protein complexes in Protein Data Bank (PDB) enables researchers to analyze the binding sites in terms of

\footnotetext{
* Correspondence: gromiha@iitm.ac.in

'Department of Biotechnology, Indian Institute of Technology Madras,

Chennai 600 036, Tamilnadu, India

Full list of author information is available at the end of the article
}

amino acid composition, preference of residues, secondary structures, solvent accessibility, electrostatic patches, hydrophobic contacts, hydrogen bonding networks and so on [1-3]. The mapping of protein-protein interactions on protein sequences suggests that hotspots can be predicted from amino acid sequences [4]. The concepts of protein-protein interactions in terms of experimental techniques, databases, organization, cooperativity and prediction of protein-protein, protein-ligand and domain interactions have been reviewed in detail earlier [5-7].

Several methods have been proposed for identifying the binding sites in protein-protein complexes based on distance between two residues [8-11]. In our earlier work, we have developed an energy based approach for defining the binding sites in protein-protein complexes 
[12]. In this work, we have analyzed the binding site residues based on sequence and structures of proteinprotein complexes. The results showed that the binding site residues have specific preferences at their vicinities and these residues are unique in protein-protein complexes. These binding site residues are more conserved than non-binding residues. In addition, several binding and non-binding residues prefer to change their conformation from helix to coil, strand to coil and coil to helix/strand. Specifically the residues Glu, Lys and Ser play important roles to conformational switching.

\section{Methods}

Dataset

We have developed non-redundant datasets of 153 protein-protein hetero dimer complexes from Protein Data Bank that have the sequence identity of less than $25 \%$ and solved with better than $3 \AA$ resolution [12]. In addition, we have used a benchmark dataset of 124 proteinprotein complexes to validate our results [13]. For comparison, we have utilized a set of 81 protein-RNA complexes [14] and 212 protein-DNA complexes [15].

\section{Identification of binding site residues}

We have calculated the interaction energy between all pairs of atoms in protein-protein complexes using AMBER force field [16]. The interaction energies of all the atoms in a residue have been summed up to assign the interaction energy of a residue. The amino acid residues with interaction energy less than $-1 \mathrm{kcal} / \mathrm{mol}$ are treated as binding site residues [17].

\section{Binding propensity}

The binding propensity $\left(\mathrm{P}_{\text {bind }}\right)$ for the 20 amino acid residues in protein-protein complexes is defined as the ratio between the frequency of occurrence of amino acid residues in the binding sites $\left(f_{b}\right)$ and in the protein as a whole $\left(f_{t}\right)$. It is calculated using the equation:

$\mathrm{P}_{\text {bind }}(\mathrm{i})=\mathrm{f}_{\mathrm{b}}(\mathrm{i}) / \mathrm{f}_{\mathrm{t}}(\mathrm{i})(1)$

where, i represents each of the 20 amino acid residues.

\section{Influence of neighboring residues}

We have analyzed the influence of neighboring residues of binding sites on various aspects: (i) " $\mathrm{B}$, where * is any residue and $B$ is a binding site residue, (ii) $B^{*}$ and (iii) ${ }^{*} \mathrm{~B} *$, which is a tripeptide with the binding site residue at the middle.

\section{Conservation score}

We have used the program AL2CO for computing the conservation score for all the residues in receptors and ligands in protein-protein complexes [17]. The target sequence has been compared with non-redundant sequences in SWISS-PROT [18] and multiple sequence alignment has been performed with ClustalW program [19]. The aligned sequences have been utilized to compute the conservation score for all the amino acid residues.

\section{Conformational switching upon complex formation}

We have computed the secondary structures of all the residues in free proteins and complexes in a set of 124 protein-protein complexes [13] using DSSP [20]. The secondary structures have been assigned as helix, strand and coil. We have analyzed the conformational changes of residues based on their locations in secondary structures, preferred amino acid residues and binding site residues.

\section{Results and Discussion}

Occurrence of amino acid residues at various ranges of interaction energies

We have identified the binding sites in protein-protein complexes based on interaction energy as explained in Methods section. We observed that $13.9 \%$ of the residues have the interaction energy of $<-1 \mathrm{kcal} / \mathrm{mol}$ and are identified as binding sites in a set of 306 proteins. We have compared the results with those obtained with distance based criteria for defining binding site residues and the data are presented in Table 1 . We noticed that only $28 \%$ residues are common to each other and the percentage of binding site residues is a balance between those identified with different cutoff distances, indicating the importance of considering the energy between different atoms to define the binding residues. In addition, $5.7 \%$ of the residues have strong repulsive energies and all these residues have been identified as binding residues in distance based criteria, which are not probable binding residues in protein-protein complexes.

\section{Conservation score for binding site residues in protein- protein complexes}

We have computed the conservation score for all the residues and noticed that the binding residues are highly conserved in protein-protein complexes. This observation is consistent with earlier studies reported in the literature [21]. We have estimated the performance of conservation score for identifying the binding sites. We found that the conservation score alone could predict

Table 1 Number and percentage of binding site residues using different methods

\begin{tabular}{lllll}
\hline Criterion & Cutoff & $\mathrm{N}_{\text {bind }}$ & $\%_{\text {bind }}$ & Reference \\
\hline Energy & $<1 \mathrm{kcal} / \mathrm{mol}$ & 5255 & 10.8 & present work \\
$C_{\alpha}$ distance & $6 \AA$ & 1972 & 4.0 & Keskin et al. [24] \\
$C_{\beta}$ distance & $6 \AA$ & 3449 & 7.1 & Glaser et al. [25] \\
Heavy atoms & $5 \AA$ & 6644 & 13.6 & Li et al. [26] \\
\hline
\end{tabular}


the binding sites at an average accuracy of $58 \%$ with a trade-off between sensitivity and specificity of $59 \%$ and $57 \%$, respectively.

\section{Binding propensity of residues in protein complexes}

We have computed the binding propensity in proteinprotein complexes and the results are presented in Figure 1. For comparison we have also included the data obtained for protein-RNA and protein-DNA complexes.

We observed that the aromatic as well as positively charged residues highly contribute to interact between the partners in protein-protein complexes, indicating the importance of cation- $\pi$, aromatic and electrostatic interactions. The comparison between protein-protein and protein-RNA complexes showed that the residues, Asp, Cys, Glu, Phe, Ile, Leu, Met, Val, Trp and Tyr have remarkably high contribution in protein-protein complexes. These residues prefer to form electrostatic, hydrophobic and aromatic interactions in protein-protein complexes. On the other hand, the residues Arg, His, Lys, Asn, Tyr, Gln and Ser highly contribute for the interaction between protein and RNA. Interestingly, these residues belong to positively charged, polar and aromatic groups, which form electrostatic, hydrogen bonds and aromatic interactions with RNA. In proteinDNA complexes, positive charged residues are more dominant than protein-protein and protein-RNA complexes. Further, polar residues prefer to interact with DNA in the form of hydrogen bonds.

Preference of tripeptides in the vicinity of binding sites We have set up the following criteria to identify the probable tripeptides for binding: (i) there should be at least three samples and the probability of being in binding sites should be more than 50\%. These conditions yielded a set of 208 unique tripeptides among 8000 possibilities. The usage of these tripeptides could predict the binding sites with an accuracy of $72.3 \%$ and the coverage of $6 \%$ of binding sites. The results for selected tripeptides are presented in Table 2.

We noticed that the central residue of all the tripeptides CWA, HHE, MNF and WFE are identified as binding site residues. The tripeptides LFP and MRR showed a preference of more than $75 \%$. Although the preference is $66.7 \%$ for ITG it has the occurrence of 12 hits and eight of them are binding site residues. The preference is significantly higher than the random choice of $1.3 \%$ (10202 binding sites and the total of 8000 tripeptides).

In Table 2, we have also included the preferred tripeptides at the binding sites of protein-RNA and proteinDNA complexes. The information on tripeptides could identify the binding sites with an accuracy of $78.7 \%$ and $71.9 \%$ in protein-RNA and protein-DNA complexes. We have compared the preferences of tripeptides at the

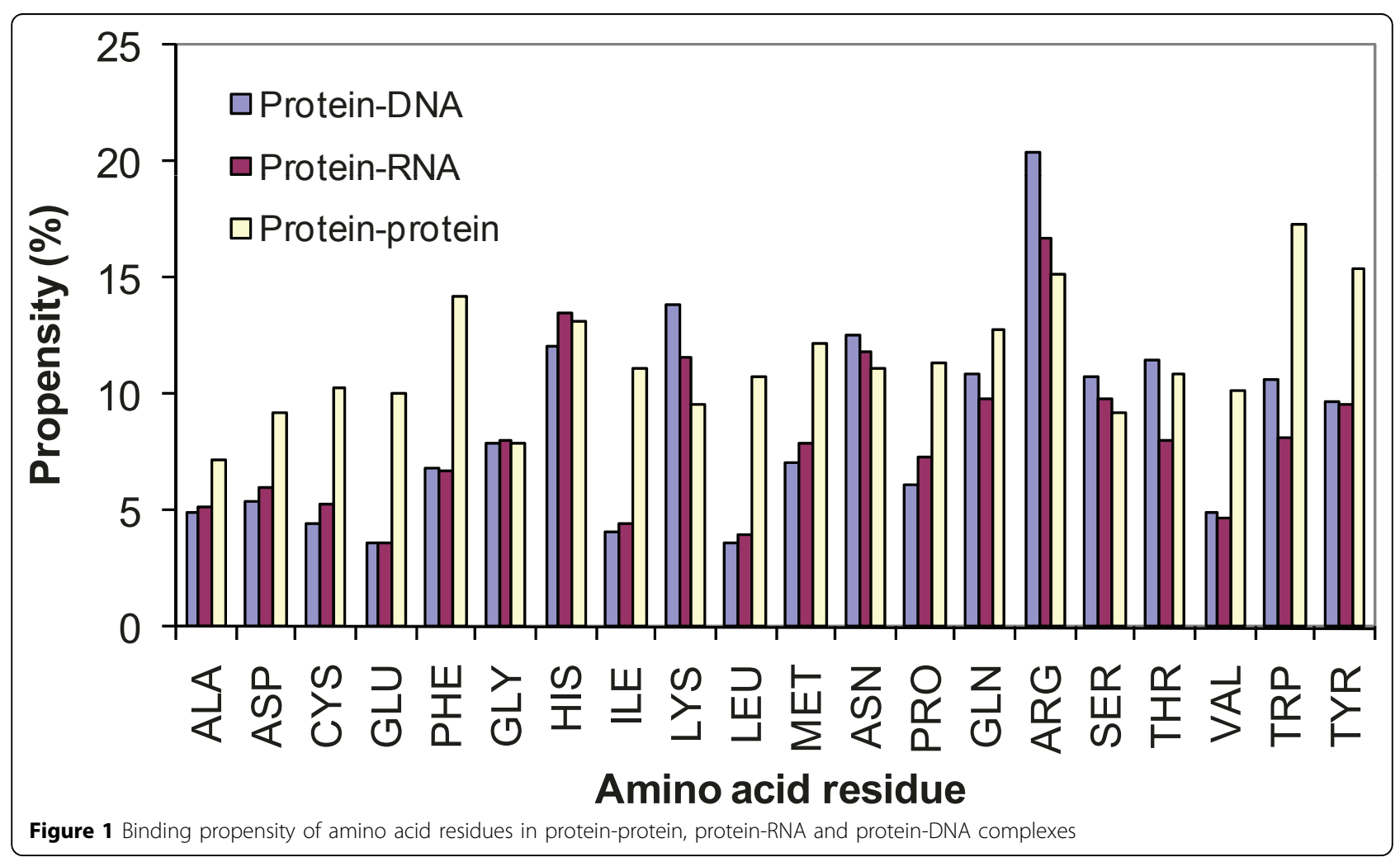


Table 2 Preferred tripeptides at the binding sites of protein-protein, protein-RNA and protein-DNA complexes

\begin{tabular}{|c|c|c|c|c|c|}
\hline \multicolumn{3}{|c|}{ Tripeptide } & $\mathrm{N}_{\mathrm{b}}$ & $\mathrm{N}_{\mathrm{t}}$ & \%bind \\
\hline \multicolumn{3}{|c|}{ Protein-protein } & & & \\
\hline CYS & TRP & ALA & 3 & 3 & 100.00 \\
\hline HIS & HIS & GLU & 3 & 3 & 100.00 \\
\hline MET & ASN & PHE & 3 & 3 & 100.00 \\
\hline TRP & PHE & GLU & 3 & 3 & 100.00 \\
\hline ILE & TYR & GLY & 8 & 12 & 66.67 \\
\hline LEU & PHE & PRO & 5 & 6 & 83.33 \\
\hline MET & ARG & ARG & 4 & 5 & 80.00 \\
\hline \multicolumn{3}{|c|}{ Protein-RNA } & & & \\
\hline GLY & TYR & GLY & 3 & 3 & 100.00 \\
\hline PRO & GLY & ARG & 3 & 3 & 100.00 \\
\hline ASP & LYS & TYR & 6 & 8 & 75.00 \\
\hline GLY & SER & THR & 3 & 4 & 75.00 \\
\hline ILE & TYR & LYS & 8 & 12 & 66.67 \\
\hline LYS & SER & ARG & 3 & 4 & 75.00 \\
\hline PRO & HIS & HIS & 3 & 4 & 75.00 \\
\hline SER & ARG & LYS & 5 & 7 & 71.43 \\
\hline VAL & GLY & SER & 6 & 8 & 75.00 \\
\hline TYR & LYS & HIS & 5 & 6 & 83.33 \\
\hline \multicolumn{3}{|c|}{ Protein-DNA } & & & \\
\hline HIS & ARG & SER & 3 & 3 & 100.00 \\
\hline SER & GLN & THR & 4 & 4 & 100.00 \\
\hline SER & TYR & GLN & 3 & 3 & 100.00 \\
\hline GLY & MET & SER & 3 & 4 & 75.00 \\
\hline GLY & ASN & ALA & 6 & 9 & 66.67 \\
\hline LYS & ARG & THR & 9 & 14 & 64.29 \\
\hline GLN & SER & TYR & 3 & 4 & 75.00 \\
\hline ARG & GLY & ASN & 5 & 7 & 71.43 \\
\hline SER & GLN & ARG & 5 & 6 & 83.33 \\
\hline SER & THR & ILE & 5 & 7 & 71.43 \\
\hline VAL & HIS & ASP & 3 & 4 & 75.00 \\
\hline VAL & LYS & CYS & 5 & 6 & 83.33 \\
\hline
\end{tabular}

$\mathrm{N}_{\mathrm{b}}$ : number of occurrence at binding sites; $\mathrm{N}_{\mathrm{t}}$ : total number of occurrence

interface of protein-protein, protein-RNA and proteinDNA complexes. Interestingly, the preferred tripeptides are unique to protein-protein complexes and none of the tripeptides are common with any of the other complexes. This result reveals the existence of different mode of recognition for the protein complexes with other biological molecules.

\section{Importance of sequence specificity revealed from dipeptide preferences}

We have analyzed the preference of residues paired with binding site residues on both $\mathrm{N}$ and $\mathrm{C}$ directions. The preferred residue pairs with $* \mathrm{~B}$ binding motifs are $\mathrm{DW}$, CW, MW, CM, CY, MR, MY, PF, PH, QW, SW, TH and $W N$. On the other hand the preferred residue pairs with $\mathrm{B}^{*}$ motifs are CW, HH, IW, MW, QM, RR, TF,
WG, WH, WM, WN, YA and YG. Further analysis on the preference of residues on $\mathrm{N}$ and $\mathrm{C}$ directions of binding sites revealed that the paired amino acids are different on both sides. Specifically, the residues at the $\mathrm{N}$ - direction of binding site Trp residue are Ala, and Asp whereas at the C-side are Met, His, Phe, Asp and Val. This result indicates the importance of sequence specificity for binding in protein-protein complexes.

We have compared the specific preferences of dipeptides in protein-protein, protein-RNA and protein-DNA complexes and the topmost five residue pairs are listed in Table 3. In this table, we included the data obtained with the motifs "B and $B *$. We observed that the residues mainly paired with $\operatorname{Trp}$ at the binding sites in protein-protein complexes. On the other hand, the residues preferred to have pairs with Arg and His in proteinRNA complexes. Interestingly, eight out of ten pairs prefer the residue Arg at the binding sites in protein-DNA complexes. This shows the different features of residue pairs at the binding sites for the proteins complexed with proteins, RNA and DNA. Further, we noticed that the residue pair Cys-Trp is common to all the three complexes, which may be a unique pair for binding.

\section{Conformational switching upon complex formation}

We have analyzed the residues that change their conformation upon binding. We noticed that approximately $7 \%$ of residues are involved in conformational changes. The analysis on different secondary structures showed that the changes between regular structures are not favorable, for example, helix to strand and vice versa. Most of the changes are associated with irregular shape or coil. We have also analyzed the preference of amino acid residues to change their conformations upon

\begin{tabular}{|c|c|c|c|c|c|}
\hline \multicolumn{2}{|c|}{ Protein-protein } & \multicolumn{2}{|c|}{ Protein-RNA } & \multicolumn{2}{|c|}{ Protein-DNA } \\
\hline \multicolumn{6}{|c|}{${ }^{*} \mathrm{~B}$} \\
\hline ASP & $\underline{\text { TRP }}$ & CYS & $\underline{\text { TRP }}$ & SER & $\underline{\mathrm{ARG}}$ \\
\hline CYS & TRP & HIS & $\overline{\mathrm{ARG}}$ & GLY & $\overline{\mathrm{ARG}}$ \\
\hline ILE & $\overline{\mathrm{TRP}}$ & ASN & $\overline{\mathrm{ARG}}$ & LYS & $\overline{\mathrm{ARG}}$ \\
\hline MET & $\underline{\text { TRP }}$ & ILE & $\underline{\text { TYR }}$ & ARG & $\underline{\mathrm{ARG}}$ \\
\hline MET & $\underline{\text { TYR }}$ & TRP & $\underline{\text { ARG }}$ & CYS & $\underline{\text { TRP }}$ \\
\hline \multicolumn{6}{|l|}{$B^{*}$} \\
\hline CYS & TRP & $\underline{\mathrm{HIS}}$ & TRP & $\underline{A R G}$ & SER \\
\hline MET & TRP & $\underline{H I S}$ & HIS & $\underline{A R G}$ & GLY \\
\hline TRP & PHE & $\underline{\mathrm{HIS}}$ & ARG & $\underline{\mathrm{ASN}}$ & TRP \\
\hline TRP & HIS & $\overline{L Y S}$ & HIS & $\overline{\mathrm{ARG}}$ & LYS \\
\hline TRP & MET & MET & TRP & $\underline{\mathrm{ARG}}$ & ASN \\
\hline
\end{tabular}

B: Binding. The binding residue are underlined. 


\section{Preference of amino acid residues for conformation change from Coil, Sheet and Helix}

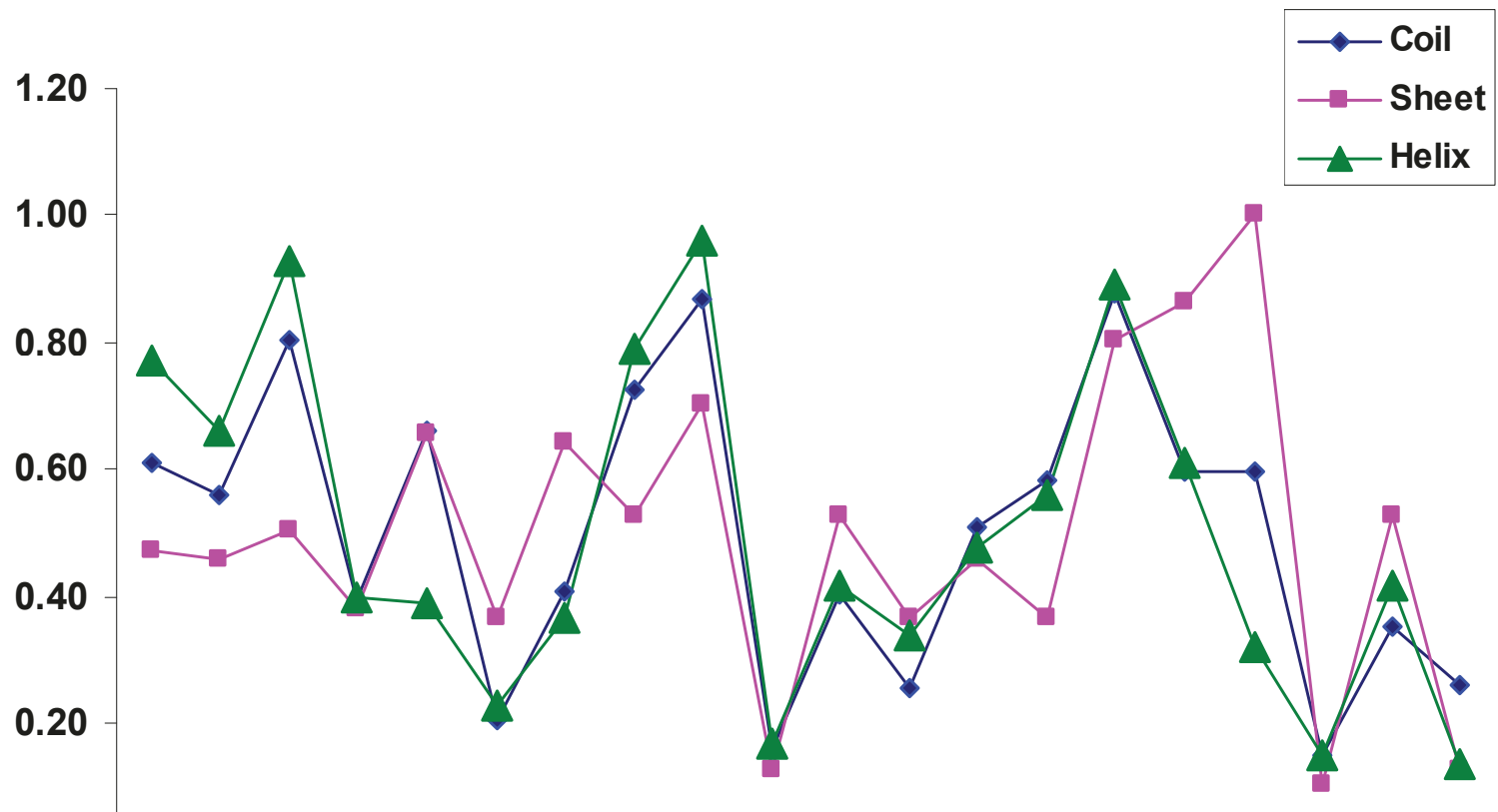

0.00

$\begin{array}{llllllllllllllllllll}A & D & E & F & G & H & \text { I } & K & L & M & N & P & Q & R & S & T & V & W & Y & C\end{array}$

Figure 2 Preference of amino acid residues for conformational change at different secondary structures.

binding in three different secondary structures. The results are shown in Figure 2.

We noticed that the residues Lys, Glu, Leu and Ser have high preference to change their conformation from helix to coil/strand as well as from coil to helix/strand. Further, Leu, Ser, Thr and Val prefer to change their conformation from strand to coil/helix. The analysis of binding site residues showed that the percentage of residues that change their conformation from coil to strand, coil to helix, strand to coil and helix to coil are, $39 \%$, $17 \%, 17 \%$ and $26 \%$, respectively. This result indicates that the proteins tend to form regular secondary structures upon binding, which agrees with the analysis that the binding site residues are located mainly in helix/ strand regions compared with coil [22]. Further, the conformation changes may be necessary for the recognition of protein-protein complexes [23].

We have also analyzed the preference of conformational changes for the neighboring residues of binding sites, $\mathrm{N}-2, \mathrm{~N}-1, \mathrm{C}+1$ and $\mathrm{C}+2$ positions. Interestingly, the preference of next residues $(\mathrm{C}+1$ and $\mathrm{N}-1$ positions) are higher than other positions and the change at the second position on both directions $(\mathrm{N}-2$ and $\mathrm{C}+2)$ are similar.

\section{Conclusions}

We have developed an energy based approach for identifying the binding sites in protein-protein complexes. The binding sites identified have been further analyzed based on different sequence and structure based parameters, conservation score, conformational switching and preference of neighboring residues. We observed that the binding residues are significantly highly conserved than the non-binding residues. We have also explored the preferences of residues at the vicinity of binding sites, which showed the importance of sequence specificity. Further, we have analyzed the importance of conformational changes upon complex formation. We noticed that the residues Ser, Leu, Lys, Glu, Thr and Val prefer to change their conformation upon binding. The information obtained in the present study will be useful for understanding and predicting the binding sites of protein-protein complexes.

\section{Acknowledgments}

This research was supported by Strategic International Cooperative Program, Japan Science and Technology Agency (JST) and Department of Science and Technology, Government of India.

This article has been published as part of Proteome Science Volume 9 Supplement 1, 2011: Proceedings of the International Workshop on 
Computational Proteomics. The full contents of the supplement are available online at http://www.proteomesci.com/supplements/9/S1.

\section{Author details}

'Department of Biotechnology, Indian Institute of Technology Madras, Chennai 600 036, Tamilnadu, India. ${ }^{2}$ Computational Biology Research Center (CBRC), National Institute of Advanced Industrial Science and Technology (AIST), 2-4-7 Aomi, Koto-ku, Tokyo 135-0064, Japan. ${ }^{3}$ Department of Bioinformatics, Bharathidasan University, Tiruchirapalli 620024, Tamilnadu, India. ${ }^{4}$ Department of Chemistry and Supercomputing Facility for Bioinformatics and Computational Biology, Indian Institute of Technology Delhi, New Delhi 110016, India.

\section{Authors' contributions}

MMG and KF designed the project. MMG carried out the computations on sequence and structural features. SS and NS are involved in conformational switching. BJ contributed in discussions.

\section{Competing interests}

The authors declare that they have no competing interests.

Published: 14 October 2011

\section{References}

1. Chakrabarti $P$, Janin J: Dissecting protein-protein recognition sites. Proteins 2002, 47:334-343.

2. Sheinerman $F B$, Honig $B$ : On the role of electrostatic interactions in the design of protein-protein interfaces. J Mol Biol 2002, 318:161-177.

3. Kortemme T, Baker D: A simple physical model for binding energy hot spots in protein-protein complexes. Proc Natl Acad Sci USA 2002, 99:14116-14121.

4. Ofran $Y$, Rost B: Protein-protein interaction hotspots carved into sequences. PLoS Comput Biol 2007, 3:e119.

5. Shoemaker BA, Panchenko AR: Deciphering protein-protein interactions. Part II. Computational methods to predict protein and domain interaction partners. PLoS Comput Biol 2007, 3:e43.

6. Keskin O, Gursoy A, Ma B, Nussinov R: Principles of protein-protein interactions: what are the preferred ways for proteins to interact? Chem Rev 2008, 108:1225-1244.

7. Shaikh SA, Jain T, Sandhu G, Latha N, Jayaram B: From drug target to leads- sketching, A physicochemical pathway for lead molecule design in silico. Current Pharmaceutical Design 2007, 13:3454-3470.

8. Sikić M, Tomić S, Vlahovicek K: Prediction of protein-protein interaction sites in sequences and 3D structures by random forests. PLoS Comput Biol 2009, 5:e1000278.

9. Fariselli P, Pazos F, Valencia A, Casadio R: Prediction of protein-protein interaction sites in heterocomplexes with neural networks. Eur J Biochem 2002, 269:1356-1361.

10. Koike A, Takagi T: Prediction of protein-protein interaction sites using support vector machines. Protein Eng Des Sel 2004, 17:165-173.

11. Ofran $Y$, Rost B: ISIS: interaction sites identified from sequence. Bioinformatics 2007, 23:e13-6.

12. Gromiha MM, Yokota K, Fukui K: Energy based approach for understanding the recognition mechanism in protein-protein complexes. Mol. Biosystems 2009, 5:1779-1786.

13. Hwang H, Pierce B, Mintseris J, Janin J, Weng Z: Protein-protein docking benchmark version 3.0. Proteins 2008, 73:705-709.

14. Gromiha MM, Yokota K, Fukui K: Understanding the recognition mechanism of protein-RNA complexes using energy based approach. Curr Protein Pept Sci 2010, 11:629-638.

15. Gromiha MM, Fukui K: Scoring Function Based Approach for Locating Binding Sites and Understanding Recognition Mechanism of ProteinDNA Complexes. J Chem Inf Model 2011.

16. Cornell WD, Cieplak P, Bayly Cl, Gould IR, Merz KM Jr, Ferguson DM, Spellmeyer DC, Fox T, Caldwell JW, Kollman PA: A second generation force field for the simulation of proteins, nucleic acids, and organic molecules. J Am Chem Soc 1995, 117:5179-5197.

17. Pei J, Grishin NV: AL2CO: calculation of positional conservation in a protein sequence alignment. Bioinformatics 2001, 17:700-712.

18. The UniProt Consortium: The universal protein resource (UniProt). Nucleic Acids Res 2008, 36:D190-195.
19. Larkin MA, Blackshields G, Brown NP, Chenna R, McGettigan PA, McWilliam H, Valentin F, Wallace IM, Wilm A, Lopez R, Thompson JD, Gibson TJ, Higgins DG: ClustalW and ClustalX version 2. Bioinformatics 2007, 23:2947-2948.

20. Kabsch W, Sander C: Dictionary of protein secondary structure: pattern recognition of hydrogen-bonded and geometrical features. Biopolymers 1983, 22:2577-2637.

21. Konc J, Janezic D: Protein-protein binding-sites prediction by protein surface structure conservation. J Chem Inf Model 2007, 47:940-4.

22. Gromiha MM, Yokota K, Fukui K: Sequence and structural analysis of binding site residues in protein-protein complexes. Int J Biol Macromol 2010, 46:187-192.

23. Lensink MF, Méndez R: Recognition-induced conformational changes in protein-protein docking. Curr Pharm Biotechnol 2008, 9:77-86.

24. Keskin O, Tsai CJ, Wolfson H, Nussinov R: A new, structurally nonredundant, diverse data set of protein-protein interfaces and its implications. Protein Sci 2004, 13:1043-55.

25. Glaser F, Steinberg DM, Vakser IA, Ben-Tal N: Residue frequencies and pairing preferences at protein-protein interfaces. Proteins 2001, 43:89-102.

26. Li W, Keeble AH, Giffard C, James R, Moore GR, Kleanthous C: Highly discriminating protein-protein interaction specificities in the context of a conserved binding energy hotspot. J Mol Biol 2004, 337:743-59.

doi:10.1186/1477-5956-9-S1-S13

Cite this article as: Gromiha et al:: Sequence and structural features of binding site residues in protein-protein complexes: comparison with protein-nucleic acid complexes. Proteome Science 2011 9(Suppl 1):S13.

\section{Submit your next manuscript to BioMed Central and take full advantage of:}

- Convenient online submission

- Thorough peer review

- No space constraints or color figure charges

- Immediate publication on acceptance

- Inclusion in PubMed, CAS, Scopus and Google Scholar

- Research which is freely available for redistribution
C Biomed Central 